\title{
Search for a cumulative neutrino signal from blazar flares using IceCube data
}

\author{
The IceCube Collaboration ${ }^{\dagger}$ \\ $\dagger$ http://icecube.wisc.edu/collaboration/authors/icrc17_icecube \\ E-mail: christoph.raab@ulb.ac.be
}

\begin{abstract}
Blazars are active galactic nuclei which have their relativistic particle jet pointing towards Earth and have been observed to emit gamma rays to very high energies. They are also candidates for the yet-unknown accelerators of ultra-high-energy cosmic rays. In such a scenario, their gamma-ray emission might be associated with neutrinos produced by hadronic interactions in the jet. Correlating the astrophysical neutrinos detected by IceCube, a cubic-kilometre neutrino telescope at the South Pole, with the gamma-ray emission from blazars could therefore reveal the origin of cosmic rays. In our method we focus on periods where blazars show an enhanced gamma-ray flux, as measured by Fermi, thereby reducing the background of the search. At the same time we test for the combined emission of a whole blazar population in a stacked search. A detection of such a neutrino flux could lead to the discovery of a source class responsible for cosmic-ray acceleration. We present sensitivities and discovery potentials for a selection of Fermi monitored sources and one year of IceCube data.
\end{abstract}

Corresponding authors: Christoph Raab and Kevin J. Meagher*1

${ }^{1}$ Université libre de Bruxelles, Science Faculty CP230, B-1050 Brussels, Belgium

35th International Cosmic Ray Conference - ICRC2017

10-20 July, 2017

Bexco, Busan, Korea

${ }^{*}$ Speaker. 


\section{Introduction}

One hundred years after the discovery of ultra-high-energy cosmic rays (UHECR), their accelerators are still unknown. On their way from the source to Earth, CR get deflected by magnetic fields and thus do not point back to their origin. High-energy neutrinos are produced in cosmic-ray interactions in the dense and energetic environment of the sources, or along their path of propagation. Thanks to their small interaction cross-section and because they have no charge, they can escape these environments and pass undeflected through magnetic fields. Therefore, neutrino astronomy is one of the most promising avenues for identifying the origin of high-energy cosmic rays [1].

IceCube is a neutrino telescope instrumenting a cubic kilometre of glacial ice at the South Pole with 5160 light-detecting modules [2]. When a neutrino interacts in the ice or bedrock, its energy and arrival direction can be reconstructed from the measured Cherenkov radiation induced by the secondary charged particles produced in the interaction. In the case of a the charged-current interaction of a $v_{\mu} / \bar{v}_{\mu}$, this yields a median angular resolution smaller than $1^{\circ}$ for energies exceeding 2 $\mathrm{TeV}[3]$.

IceCube has detected a diffuse flux of astrophysical neutrinos of that same flavour [4], but so far no spatial clustering around any particular point source. For IceCube to detect such a clustering by distinguishing individual sources from any source population, its density $\rho$ must be sufficiently small. On the other hand, a sufficient luminosity $L$ is required if a source class should have an emission per volume $L \cdot \rho$ that contributes significantly to the astrophysical flux [5] [6].

As can be seen in [5], blazars do fulfil both these criteria. They are also known to be highly variable sources of gamma rays. The gamma rays on their own however do not prove cosmic-ray acceleration, since they could be leptonic or hadronic in origin. Several models predict hadron acceleration by internal shocks in blazar jets and predict emission of both gamma rays and highenergy neutrinos [7]. The discovery of a correlation between gamma-ray flares and a neutrino flux would imply at least a partially hadronic production mechanism of the observed gamma rays, and thus identify blazars as cosmic-ray accelerators.

Following the arguments in [5], the low source density of blazars also means that if they were responsible for the entire astrophysical neutrino flux, the closest one should already have been detected as a point source. A stacking analysis of $149 \mathrm{HBL}^{1}$ blazars from the 2FHL catalogue [8] excludes them from emitting more than $4.5 \%-5.7 \%$ of the astrophysical $v_{\mu} / \bar{v}_{\mu}$ flux at $90 \%$ C.L.. However, if their emission in neutrinos is concentrated in flares like it is for gamma rays, an analysis that exploits this extreme variability might still detect a flux whose time integral is below this limit.

Here we present a search for high-energy neutrinos detected by IceCube correlated in time with the combined gamma-ray flux measured by the Fermi Large Area Telescope (LAT) from a set of blazars. Contrary to previous analyses which focused on individual flaring sources [9], we apply a stacking approach (see [10] for details on the stacking technique), which improves our sensitivity to a weak signal.

\footnotetext{
${ }^{1}$ high synchrotron peaked BL Lac
} 


\section{Analysis Method}

\subsection{Likelihood}

Our analysis is based on a maximum likelihood ratio method, as has been used before in timeintegrated searches for single point sources (e.g. [3]). The signal hypothesis represented in this likelihood depends on the position, strength and spectral index of a source's emission. For our search focused on flares, we augment the signal hypothesis for a source $(k)$ by a time distribution $\mathscr{T}^{(k)}(t)$. We derive this from Fermi-LAT light curves (see sec. 3 ) by applying a flux threshold $\Phi_{0}^{(k)}$ and normalising the result. The likelihood then considers events where the flux is below threshold as entirely background-like.

$$
\mathscr{T}^{(k)}\left(t \mid \mathrm{LC}_{k}, \Phi_{0}^{(k)}\right)=\max \left\{\left(\operatorname{LC}_{k}(t)-\Phi_{0}^{(k)}\right) / \text { norm }, 0\right\}
$$

In previous time-dependent analyses such as [9], this threshold was left as a free parameter between 0 and the maximum of the light curve to reflect the fact that we expect enhanced neutrino emission to occur during the flaring states, but to an unknown degree. At a threshold of 0 , all the gamma-ray emission would be related to neutrino emission and thus directly proportional.

In a stacked search as described in [10], the global signal hypothesis is a sum of the local hypotheses for a catalogue of sources. We assume these to have identical spectral indices and source strengths proportional to the global strength by a normalised weight $w^{(k)}$ :

$$
S(x, E \mid \gamma)=\sum_{k \in \text { sources }} w^{(k)}(\gamma) \mathscr{S}^{(k)}(\gamma)
$$

Here, $w^{(k)}$ expresses any assumptions about the relative brightness of each source's neutrino flux as well as the detector response at their respective declinations.

We choose to fix the spectral index in order to avoid an increased effective trial factor and numerical challenges. This can be partially justified by an analysis of bright blazar flares [11] which shows their spectral indices to be constrained to 1.9-2.6, with flares longer than $\sim 1$ day converging to $2.3-2.4$.

When applying this principle to a time-dependent analysis, we can make the equivalent argument for the flux threshold and assume that no source produces neutrinos during its quiescent period. We express this as a fixed threshold $\left\{\Phi_{0}\right\}_{\text {est. }}^{(k)}$ estimated for each light curve individually, to accommodate their different brightness and variability characteristics.

$$
S_{i}(x, E, t \mid \gamma)=\sum_{k \in \text { sources }} w^{(k)}(\gamma) \mathscr{S}^{k}\left(x, E, t \mid \gamma,\left\{\Phi_{0}\right\}_{\text {est. }}^{(k)}\right)
$$

We perform this threshold estimation starting from the denoised Bayesian block representation of the light curve (see sec. 3). We take the derivative, i.e. for each adjacent block the difference between their fluxes divided by the distance of their centres $\left(\Phi_{i+1}-\Phi_{i}\right) /\left(t_{i+1}-t_{i}\right)$. By taking the time-weighted median of this derivative, we can select one half of the observed time where the light curve is locally most steady, largely independent of the overall variability of the light curve. We then use fluxes measured during this time to compute a mean flux. As the goal is to exclude low and 


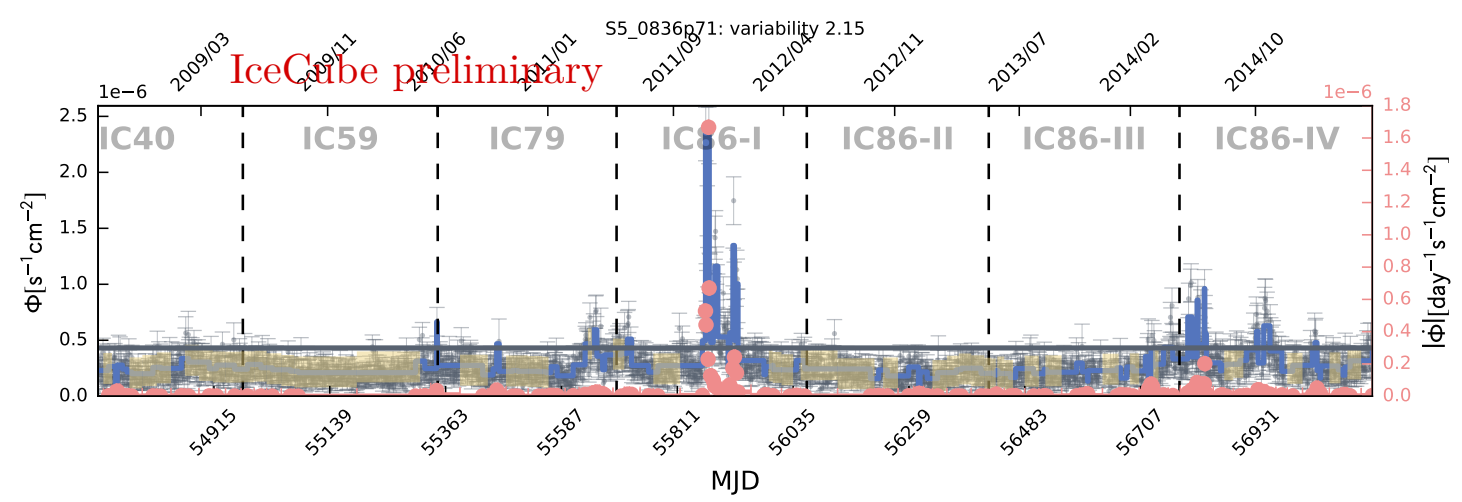

Figure 1: A light curve of the flat spectrum radio quasar S5 $0836+71$, covering the observation periods of the IceCube detector in its 40-string configuration, up to the fourth year of the completed detector with 86 strings (IC86-IV). The grey data points show the gamma-ray flux observed by the Fermi-LAT. The blue curve shows its denoised block representation. The time derivative between each two blocks is drawn as red points (right axis). We consider the time periods when the derivative does not exceed its median as quiescent (yellow bands). The mean flux during these time periods and the standard deviation determine the flux threshold (grey line).

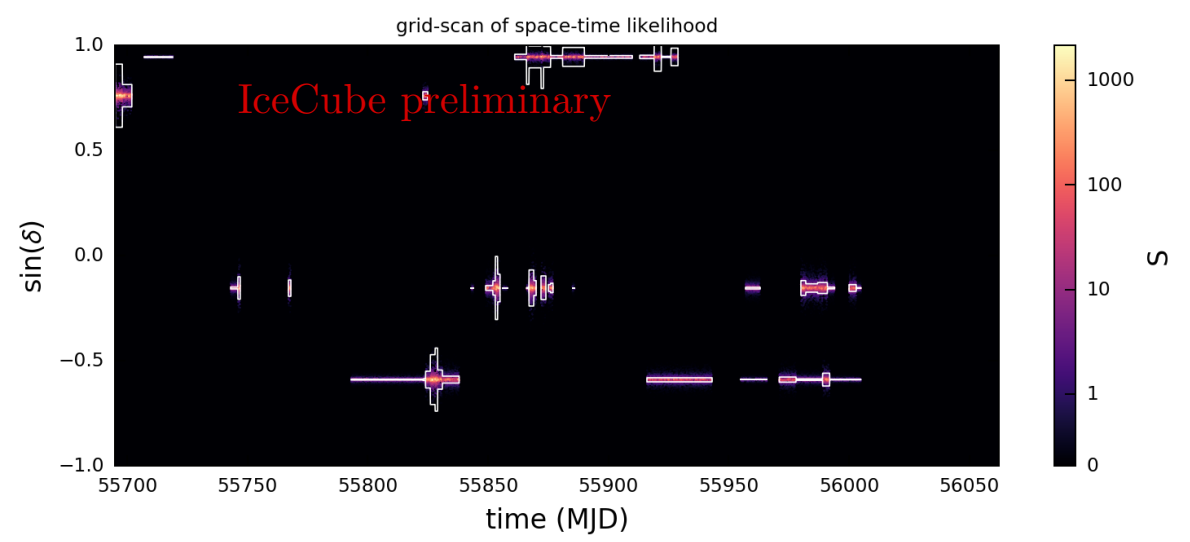

Figure 2: Illustration as the likelihood as a function of space and time for four sources, evaluated for a grid scan of the declination and time, maximised in right ascension. The time distributions for each source are drawn for comparison as white lines with an arbitrary scale.

steady fluxes in a robust way, we take the final threshold as this mean steady flux plus five times it root mean square deviation from the mean. We illustrate this procedure in figure 1. Figure 2 shows the space-time likelihood, i.e. the full likelihood without energy, evaluated for illustration on a grid scan given four arbitrary sources. Events which have a lower time likelihood need to be closer to the corresponding source in order to be treated equally.

\subsection{Performance}

In order to evaluate the sensitivity of the $\log \mathscr{L}$-derived test statistic to the addition of signal 
events to pure-background data we use a toy Monte Carlo that generates background and signal events.

For the pure background case, we do this by choosing a random time for each event from within the uptime where the detector was taking data. These are equivalent, since seasonal variations do not play a role in the analysed sample [12]. For a neutrino entering the detector at the reconstructed local direction, and at the new, random time, we compute the new direction in equatorial coordinates. We keep all other event information, so that the data still describe the observed detector response. We call this procedure time scrambling, and it has been used previously in [9].

To generate signal data with an expected number of signal neutrinos $\mu$, each realisation is a mixture of a background time scrambling and a number of simulated events following a Poisson distribution of mean $\mu$. To simulate the point spread function, we consider Monte Carlo events from within a declination band for each source, and then rotate them so their true position is at the source. We draw events so that their energies follow a power-law flux (we use $E^{-2}$ as a benchmark) from the sources in consideration. We also take the relative effective area of the detector at the respective declinations into account. We then assign every event belonging to a certain source a random time, again within the uptime but now weighted according to the time p.d.f. used for this source (we use the same as in the likelihood, see sec. 2.1).

Ultimately we can evaluate the performance of the method by determining the $\mu$ at which the power $\beta=0.5$ of the hypothesis test corresponds to a certain significance. Specifically we determine the median $5 \sigma$ discovery potential with $\alpha=2.87 \times 10^{-7}$ by extrapolating the backgroundonly test statistic distribution (see fig. 3).

\section{Fermi Data and Source Selection}

The analysis method described above requires continuous light curves for all sources. We therefore use data from the Fermi Large Area Telescope, which scans the entire sky every $3 \mathrm{~h}$ [13]. The Fermi Monitored Source List ${ }^{2}$ comprises 148 sources which at the time of writing have experienced a strong flare during the Fermi mission. We use the SIMBAD astronomy database ${ }^{3}$ to remove non-AGN objects and assume that the rest are blazars. This includes eight sources not directly identified by SIMBAD of which three have been identified as blazars in literature: PKS 1510-08 9 [14], S5 0716+714 4 and 0FGL J0910.2-5044 [15]. This leaves 125 sources to be analysed for their variability. In the final analysis we will use source classification from the third Fermi catalogue, $3 \mathrm{FGL}^{5}$.

For each of these sources we derive the gamma-ray light curves with aperture photometry using the Fermi Science Tools v10r0p $5^{6}$. We take photons above $100 \mathrm{MeV}$ in a $2^{\circ}$ radius around the source location. This radius corresponds approximately to the $68 \%$ containment radius at $200 \mathrm{MeV}$. We correct for the loss of photons outside the analysis region, an effect which decreases with energy [13]. We apply further selection criteria and processing according to [12] and

\footnotetext{
${ }^{2}$ https://fermi.gsfc.nasa.gov/ssc/data/access/lat/msl_lc/

${ }^{3}$ http: //simbad.u-strasbg.fr/simbad/

${ }^{4}$ http $: / /$ tevcat . uchicago.edu/?mode=1\&showsrc $=172$

5 https://fermi.gsfc.nasa.gov/ssc/data/access/lat/4yr_catalog/

${ }^{6}$ https://fermi.gsfc.nasa.gov/ssc/data/access/lat/
} 


$\begin{array}{lllll}0716+714 & 4 \mathrm{C}+28 \mathrm{~d} 07 & \text { OG 050 } & \text { PKS 0521-36 } & \text { S5 1803+78 } \\ 1150+497 & \text { B2 1520+31 } & \text { OJ 287 } & \text { PKS 2247-131 } & \text { TXS 1530-131 } \\ 1510-089 & \text { BL Lac } & \text { PKS 0402-362 } & \text { PMN J1038-5311 } & \\ 1633+382 & \text { CTA 102 } & \text { PKS 0454-234 } & \text { PMN J2345-1555 } & \\ \text { 3C 279 } & \text { NRAO 676 } & \text { PKS 0502+049 } & \text { S5 0836+71 } & \end{array}$

Table 1: Preliminary list of sources selected for the analysis.

count the photons in 1-day bins. After removing days without observations or low exposure, we then denoise the daily measurement using the Bayesian block algorithm [16] which seeks to combine those sequences of bins which are compatible with a constant emission. We also follow [12] in selecting the most variable light curves during the analysis period. The cut is described by

$$
\frac{\Phi_{\max }-\Phi_{\min }^{(80)}}{<\Phi^{(80)}>}>1
$$

where $\Phi_{\min }^{(80)}$ is the minimum flux within the central $80 \%$ of blocks, and $\left\langle\Phi^{(80)}\right\rangle$ is the mean flux calculated over the same set. Since the Fermi Monitored Source List ${ }^{7}$ is selected by applying a threshold of $10^{-6} \mathrm{~cm}^{-2} \mathrm{~s}^{-1}$ to the observed flux without an absolute flux calibration, we also reapply this cut to the calibrated fluxes. We therefore end up with a subset of 22 from the sources used in [12], which are listed in table 1.

\section{Discovery Potentials}
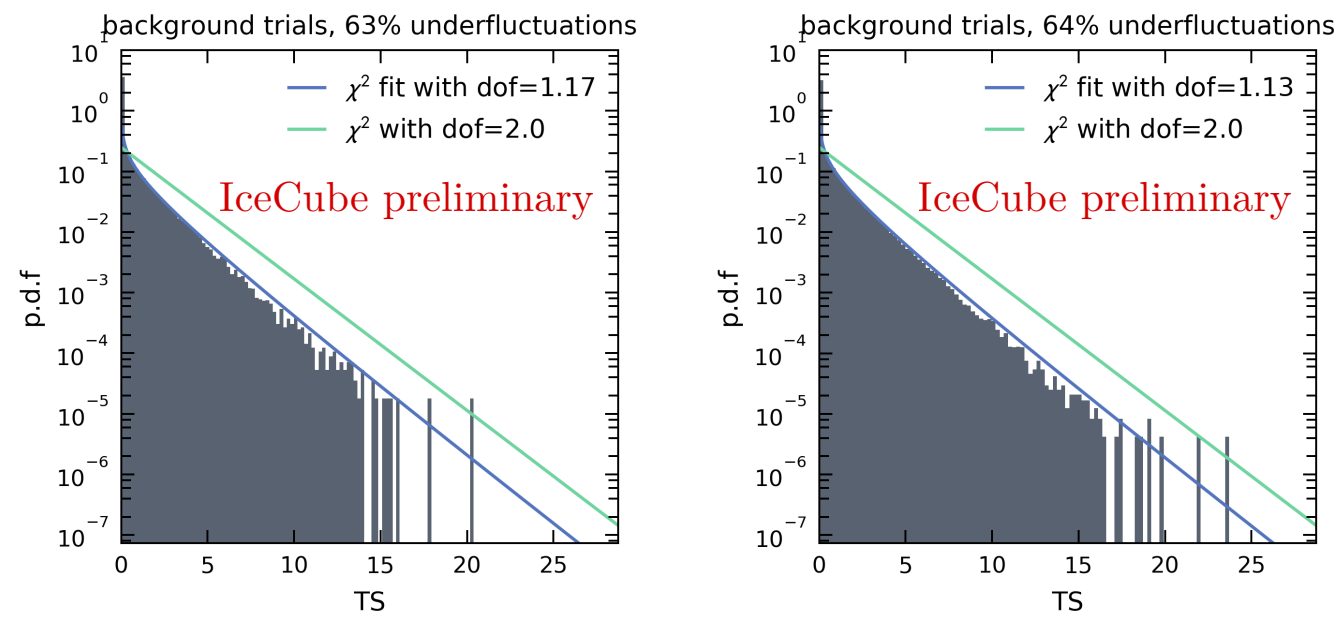

Figure 3: Distribution of the test statistic for trials on realisations of the background-only hypothesis (grey), with (left) and without (right) relative source weights in the likelihood. To compute the discovery potentials, we extrapolate with the fit $\chi^{2}$ distribution (blue line); we show the classical assumption of $n_{\text {dof }}=2$ for comparison.

\footnotetext{
${ }^{7}$ https://fermi.gsfc.nasa.gov/ssc/data/access/lat/msl_lc/
} 
We use the same sample of events as [3] during the first season of IceCube data-taking in its full configuration. In figure 4 (left) we show $5 \sigma$ median discovery potentials for an individual source while varying the threshold of the light curve. This shows how the discovery potential depends on the amount of background that is removed by the time-dependency; assuming a higher threshold, fewer events are needed. In the stacked case, we instead predefined the threshold using the light curve data (see sec. 2.1). In the following we use two different signal hypotheses. First, we assume that the neutrino flux is proportional to the gamma-ray flux above the threshold, and weight the likelihood for each source according to the integrated gamma-ray flux. This reflects the case where for each source the fraction of hadronically produced gamma rays is equal. Second we assume equal weighting such that each source has the same neutrino fluence. The resulting background test statistic distributions and their respective extrapolations are shown in 3. The effective degrees of freedom are now closer to 1 than to 2, as the fit shows. For each signal hypothesis we use the respective model in the signal injection, which is the most optimistic case, to arrive at the discovery potentials in figure 4 (right). There, we have done this calculation not only for the full source list, but also for any subset of the $N$ brightest sources, according to the integrated gamma-ray flux. We show that many sources that are individually undetectable can sum up to a discovery in the stacking. Typically we can expect a discovery for stacking 10 sources if $\sim 1$ neutrino is detected from each.
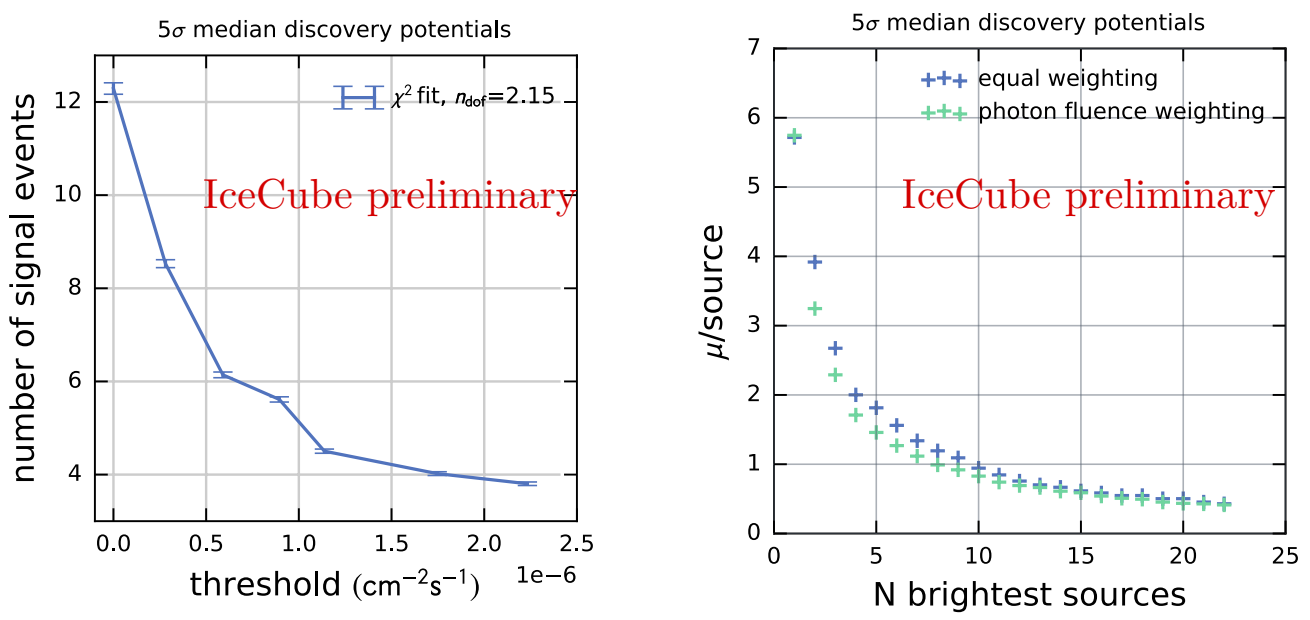

Figure 4: Left: $5 \sigma$ median discovery potentials in terms of detected neutrinos per source, for a single source, varying the flux threshold in the definition of the time distribution (see eq. 2.1). Right: The same for several possible combinations out of the total 22 sources by taking the $N$ sources with the highest fluence integrated above the light curve threshold, for $N \in\{1, \ldots, 22\}$. Points in blue assume the neutrino emission is equal for each source, and points in green assume it is proportional to the same total fluence as used for the ordering.

\section{Conclusions and Outlook}

We have shown that our implementation of the method fulfils its goals with respect to reducing background via time-dependence, and increasing sensitivity via stacking. Nevertheless, several 
enhancements are possible both for the method itself and its application. The former includes an improved extrapolation of the TS distribution that gives less conservative discovery potentials than the currently used fit, a more realistic estimation of the thresholds, with a possible common parameter analogous to figure 4 (left). For the weights, the existing assumptions could be more closely motivated by the expected hadronic character of the source according to astrophysical arguments. Alternatively we could fit the weights, which in the case of non-detection might improve the limits provided the expected weighting varies by orders of magnitude. We further need to characterise the biases of the $\log \mathscr{L}$ fit, especially to allow interpretation in case of a discovery. In either case, we should ultimately express the number of signal events in terms of a flux in order to allow comparisons to astrophysical models as well as other analyses.

Once the analysis method is finalised we will apply it to all years of available IceCube data. This will in turn lead to a longer source list. We can then apply more stringent criteria on the object type, using other catalogues such as $3 \mathrm{FGL}^{8}$, to facilitate interpretation of limits or discoveries. To the same end, we could split up the source list into subsets according to their weight. Their light curves should be derived using likelihood analysis instead of aperture photometry. Then astrophysical models can be used where appropriate to give the neutrino time profile. Finally, it is important to keep in mind that so far we are using IceCube data optimised for time-integrated searches. It is possible that a data sample with a higher background contamination but also higher signal efficiency will prove more suitable.

\section{References}

[1] M. Kowalski, arXiv:1411.4385 [astro-ph.HE] (2014).

[2] IceCube Collaboration, M. G. Aartsen, et al., JINST 12 (2017) P03012.

[3] IceCube Collaboration, M. G. Aartsen, et al., Astrophys. J. 835 (2017), 2151.

[4] IceCube Collaboration, M. G. Aartsen, et al., Astrophys. J. 833 (2016), 13.

[5] P. Mertsch, M. Rameez, and I. Tamborra, JCAP 1703 (03, 2017).

[6] P. Lipari, Phys. Rev. D78 (2008), 083011.

[7] W. Essey, et al., AAS Meeting 224 (2009), 407.06.

[8] IceCube Collaboration, PoS ( ICRC2017) 994 (2017).

[9] IceCube Collaboration, M. G. Aartsen, et al., Astrophys. J. 807 (2015), 46.

[10] IceCube Collaboration, M. G. Aartsen, et al., Astrophys. J. 835 (2017), 145.

[11] K. Nalewajko, MNRAS 430 (2012), 2 1324-1333.

[12] A. Christov. PhD thesis, Université de Genève, 11/01, 2016. ID: unige:91766.

[13] Fermi Collaboration, W. B. Atwood, et al., Astrophys. J. 697 (2009) 1071-1102.

[14] D. C. Homan, et al., Astrophys. J. 580 (12, 2002) 742-748.

[15] N. Mirabal, arXiv:0908.1389 [astro-ph.HE] (2009).

[16] J. D. Scargle, et al., Astrophys. J. 764 (02, 2013) 26 pp. ID 167.

\footnotetext{
${ }^{8}$ https://fermi.gsfc.nasa.gov/ssc/data/access/lat/4yr_catalog/
} 